\title{
DUETs and priority setting
}

\author{
Derek Richards \\ Centre for Evidence -based Dentistry, Oxford \\ DUETs is the UK Database of Uncertainties about the Effects of Treatments (UKDUETs) \\ DUETs is available on the NHS Evidence website at www.library.nhs.uk/duets/
}

There are many uncertainties in life and sadly there seem to be many uncertainties about the effects of a wide range of treatments. The rise of the systematic review and the work of the Cochrane collaboration have led to a large increase in both the number and the quality of systematic reviews in a wide range of health care disciplines. While many of these reviews do provide answers to the questions posed, many of them do not. Many of these unanswered questions relate to issues that matter most to patients and clinicians. It is because of this that the DUETs database gives priority to presenting unanswered questions about the effects of treatments, which have been asked by patients and clinicians, while also noting therapeutic uncertainties identified through systematic reviews, clinical guidelines and other formal mechanisms.

The Oral and Dental Conditions section of the database currently contains 56 records. The majority of these relate to known uncertainties identified as a result of the findings from Cochrane reviews. An example of a record relating to the surgical removal of asymptomatic third molars is shown in Figure 2.

Recognising those uncertainties is one thing, addressing them is another. The researchers themselves often drive the research agenda and there has often been a tendency to overlook unanswered questions about the effects of treatments that are of importance to patients and clinicians.

In order to address this the James Lind Alliance (JLA) has been established (http://www.lindalliance.org/) to bring together patients, carers and clinicians to help identify and prioritise the top ten uncertainties or unanswered questions in a range of clinical areas. The JLA has produced a guidebook on how to develop priority setting partnerships (http://www.jlaguidebook.org/). The JLA is currently working with a cleft lip and palate priority setting partnership to develop their list of the top ten unanswered questions.

The DUETs database and JLA are synergistic, and with increasing moves towards patient reported outcomes, patient involvement in research and other moves to place the patient's voice further up the agenda the role of the DUETs database and the JLA looks set to grow.

Evidence-Based Dentistry (2012) 13, 92

doi:10.1038/sj.ebd.6400883

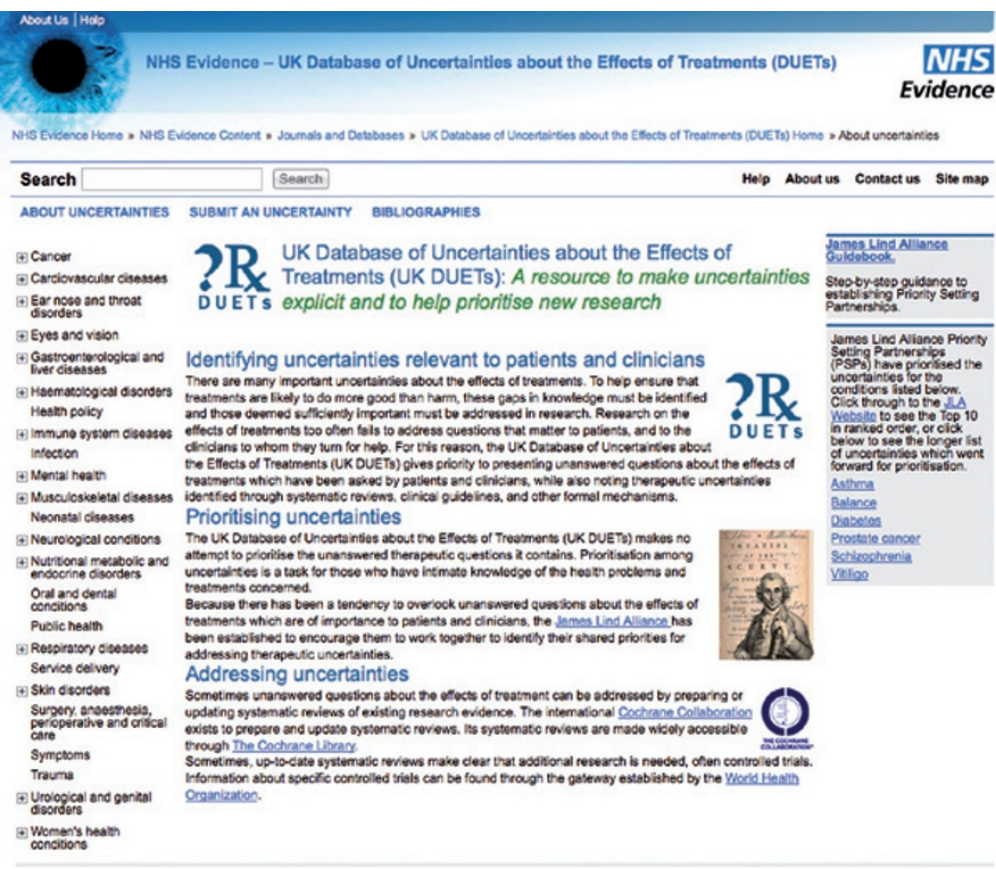

Fig. 1 The DUETs database home page

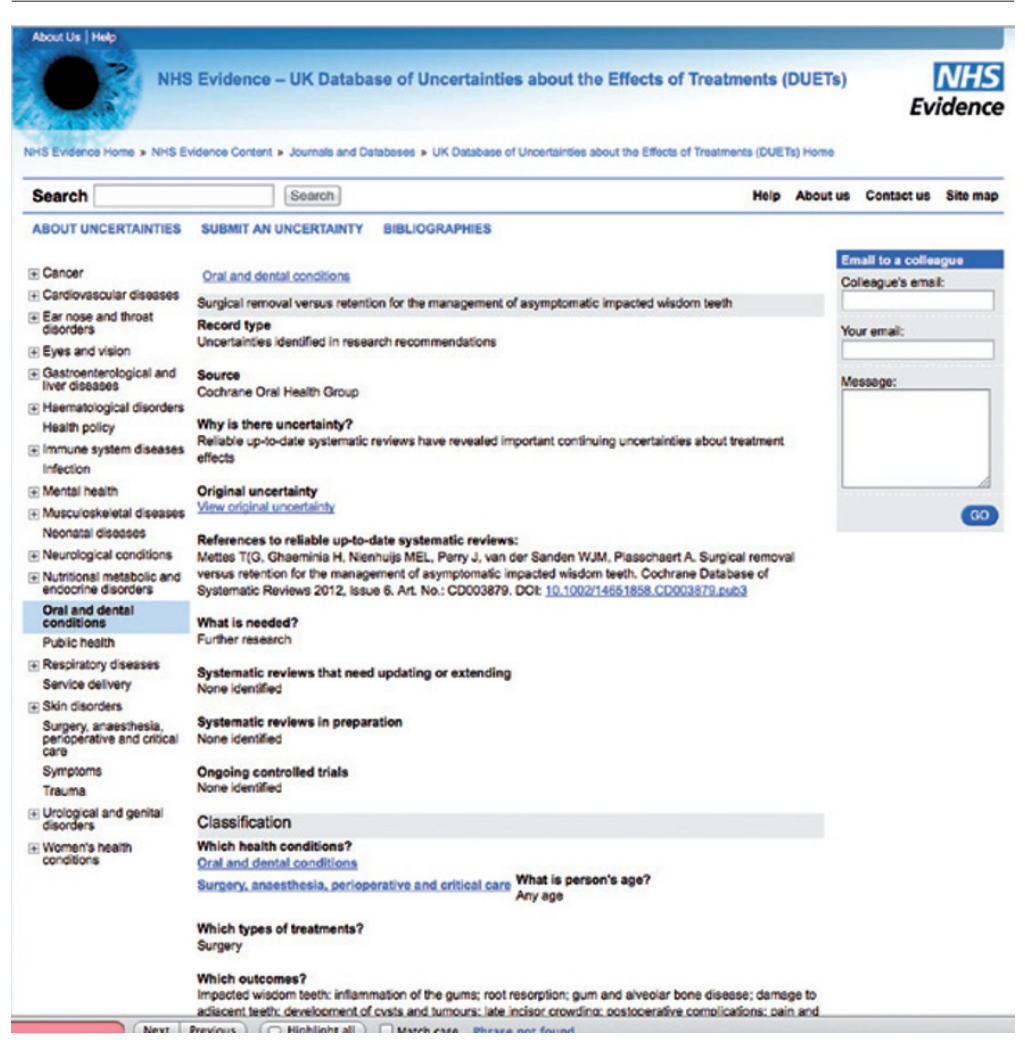

Fig. 2 Example page from the DUETs database: Surgical removal versus retention for the management of asymptomatic impacted wisdom teeth 